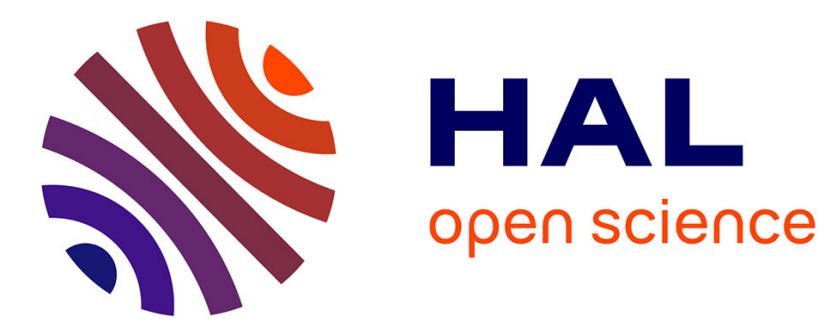

\title{
Catégorisation de comment subordonnant
}

Florence Lefeuvre

\section{To cite this version:}

Florence Lefeuvre. Catégorisation de comment subordonnant. Travaux de linguistique, Bruxelles, 2009, p. 63-88. halshs-01142252v2

\section{HAL Id: halshs-01142252 \\ https://shs.hal.science/halshs-01142252v2}

Submitted on 18 Apr 2015

HAL is a multi-disciplinary open access archive for the deposit and dissemination of scientific research documents, whether they are published or not. The documents may come from teaching and research institutions in France or abroad, or from public or private research centers.
L'archive ouverte pluridisciplinaire HAL, est destinée au dépôt et à la diffusion de documents scientifiques de niveau recherche, publiés ou non, émanant des établissements d'enseignement et de recherche français ou étrangers, des laboratoires publics ou privés. 


\section{Catégorisation de comment subordonnant ${ }^{1}$}

Florence Lefeuvre

Université de Paris-III

LaTTiCe, UMR 8094

\section{A citer sous cette forme: Lefeuvre F., 2009: "Catégorisation de comment} subordonnant ", Travaux de linguistique, Etudes sur comment (Moline E. ed.), $\mathrm{n}^{\circ}$ 58, $\mathbf{p}$.

\section{3-88.}

L'objet de cet article est d'examiner si comment subordonnant ${ }^{2}$ se réduit à un emploi de percontatif interrogatif ou s'il connaît des emplois de percontatif exclamatif, d'intégratif voire de complétif. Notre cadre théorique est issu de Le Goffic 1993, 2002. Selon cet auteur, quatre types de subordonnées « saturent un cadre logique » (Le Goffic 1993), la percontative interrogative ou exclamative (interrogative ou exclamative indirecte), l'intégrative (relative sans antécédent et circonstancielle en $q u$ - ou $s i$ ), la relative (à antécédent nominal) et la complétive. Comment est parfois présenté en distribution complémentaire avec comme (cf. Léard 1992) [« forme autonome»vs «forme non autonome »]. Ce type de complémentarité concerne d'autres mots en $q u$-, notamment le célèbre couple que / quoi ${ }^{3}$. Nous en avons montré les limites dans Lefeuvre 2006a. La répartition syntaxique de comme et comment, supposée claire, a pu donner lieu à la composition d'un test afin de catégoriser les subordonnées dans les cas litigieux (cf. par exemple Le Goffic 1987). Nous verrons dans cet article que comment connaît des emplois généralement attribués à comme (dans les cas de percontatif exclamatif et d'intégratif), ce qui relativise l'efficacité de ce test.

Comment fait partie des mots en $q u{ }^{4}$ qui peuvent s'analyser comme des variables. Cela se traduit par l'impossibilité pour comment de survenir au sein d'une assertion :

\footnotetext{
${ }^{1}$ Je remercie E. Moline et les reclecteurs pour leurs remarques. Je reste seule responsable, bien entendu, de ce qui suit.

${ }_{2}^{2}$ Nous nous en tiendrons ici à la définition suivante du subordonnant: introducteur non assertable d'une proposition qui est enchâssée dans une proposition matrice. Le mot de «percontatif » permet de distinguer les interrogatives directes des interrogatives indirectes sans supposer de lien entre les deux types de proposition. Dans le même ordre d'idée, cf. Moline soumis a et b qui préconise l'appellation de «phrase à interprétation interrogative » plutôt que celle d'interrogative indirecte. Pour des arguments en faveur du caractère subordonnant du mot en $q u$ - dans les percontatives, cf. Lefeuvre 2006a et Krazem 2007.

${ }^{3}$ Cf. par exemple Que/*quoi veux-tu? / Tu veux quoi / *que? Pour l'étude de ce problème, cf. par exemple, Hirschbühler, 1987 et, pour un récapitulatif, Lefeuvre 2006 a.

${ }^{4}$ L'ensemble des mots en qu- comprend les pronoms qui, que, quoi, lequel, le pronom ou déterminant quel, les adverbes quand, comme, comment, combien, où, que (Que c'est beau!), pourquoi (à partir de quoi). Il s'agit
} 
[1] * Je peux le joindre comment.

Seule l'interrogative est possible :

[2] Je peux le joindre comment?

Tout comme les autres mots en $q u$ - (cf. Lefeuvre 2006a), l'adverbe ${ }^{5}$ comment est susceptible de connaître 3 types d'emplois.

i) Il peut être interrogatif :

[3] Comment trouves-tu ma robe ? (Garat)

ii) subordonnant, principalement dans les percontatives interrogatives en français contemporain :

[4] Je ne sais pas comment elle s'y prend pour le repérer (Winckler)

iii) non subordonnant et employé en périphérie de la proposition assertée (cf. Lefeuvre et Pierrard 2008), lorsqu'il est assorti d'une proposition subordonnée, en tant qu'indéfini :

[5] En tous affaires, quand ils sont passés, comment que ce soit, j'y ay peu de regret (16 ${ }^{\mathrm{e}} \mathrm{s}$, Montaigne, ex. tiré de Gougenheim 1984)

Comme a connu également ces emplois :

[6] Comme est-ce qu'il s'appelle ? (Molière ; ex. tiré de Fournier 1998)

[7] Vous a-t-on dit comme on le nomme ? (Molière ; ex. tiré de Fournier 1998)

[8] Vous plustost par vos mains, comme que soit, cette vie enlevez $\left(16^{\mathrm{e}} \mathrm{s}\right.$, Des Masures, ex. tiré de Gougenheim 1984)

Pour cette étude, nous nous sommes appuyée sur un corpus de 401 exemples de comment subordonnant tirés de la base Frantext de 1998 à 2007.

Nous défendrons ici l'hypothèse que comment subordonnant, issu d'emplois variés qui recoupaient largement ceux de comme en ancien français, connaît une diversification catégorielle, oscillant entre des emplois de percontatif interrogatif, de percontatif exclamatif, d'intégratif, voire de complétif. Il existe un nombre d'emplois non négligeable (50 exemples sur 401) où il est difficile de catégoriser comment. Cela dit, notre travail sur corpus indique que c'est le premier emploi — celui de percontatif interrogatif - qui est largement majoritaire. Nous examinerons tout d'abord, les emplois de comment percontatif interrogatif,

d'une liste fermée qui joue un rôle essentiel dans l'interrogation et la subordination. Ces termes remontent à un thème $* K^{w}$ indo-européen (Meillet \& Vendryes, 1960) qui se caractérise par sa valeur d'indéfini.

${ }^{5}$ Nous considérerons comment comme un adverbe, équivalent à un groupe prépositionnel. Il est possible également de l'analyser comme une proforme, mot qui a l'avantage de dépasser la dichotomie pronom / adverbe et de montrer la ressemblance de fonctionnement entre les mots en $q u$ - qui jouent un rôle essentiel dans la subordination et l'interrogation (cf. Hadermann 1993). 
puis successivement ceux de comment percontatif exclamatif, de comment intégratif et de comment complétif.

\section{Comment percontatif interrogatif ( 351 exemples)}

Après avoir examiné les critères de reconnaissance des percontatives interrogatives, nous les appliquerons aux exemples de notre corpus.

\subsection{Critères de reconnaissance des percontatives interrogatives}

Nous allons voir ci-dessous les trois principaux critères qui permettent de reconnaître les percontatives interrogatives en comment.

i) Certains mots en $q u$ - distinguent leur emploi percontatif interrogatif de leur emploi intégratif. Cette dissemblance est manifeste avec qu'est-ce que qui peut s'employer dans un registre familier avec des percontatives interrogatives :

[9] Je me demande qu'est-ce qu'il devient alors qu'il ne le peut pas avec des intégratives, sauf dans un registre très familier (cf., pour ce test, Pierrard $1988: 32)$ :

[10] Je fais qu'est-ce que je veux.

Nous pourrons appliquer cette possibilité d'emploi comme test pour reconnaître la nature de la proposition en comment $P$ : la possibilité de paraphraser l'exemple par comment est-ce que indique qu'il s'agit d'une percontative :

[11] Je me demande comment il fait

[12] Je me demande comment est-ce qu'il fait

Quel, quant à lui, ne peut apparaître que dans des emplois percontatifs (Le Goffic, 1987 et Hadermann 1993) :

[13] J'aimerais savoir à quel individu il faisait référence.

[14] * J'ai rêvé hier à quel individu

ce qui nous permet, par rapport à comment, de proposer un test avec de quelle façon qui comprend le déterminant quel et un nom renvoyant au sémantisme de la manière. Comment percontatif interrogatif :

[15] Mon désarroi se transforma en triomphe quand, de retour à mon hôtel (je me demande encore comment j'y suis parvenu), je pus me saisir en tâtonnant d'une ancienne paire de lunettes restée, par miracle, au fond de ma valise. (Pontalis) peut se paraphraser par de quelle façon: 
[16] Je me demande encore de quelle façon j’y suis parvenu.

Retenons toutefois que cette paraphrase s'avère difficile à appliquer lorsque comment est de type qualitatif, dans des énoncés où le noyau de comment $P$ est le verbe être :

[17] Ne vous inquiétez pas : il est chez moi. J'espère que vous n'êtes pas trop malheureuse. Vous savez comment il est ! (Buron)

[18] * Vous savez de quelle façon il est

ou le verbe aller :

[19] Je me demande comment il va

[20] * Je me demande de quelle façon il va

L'emploi du verbe faire, possible avec comment sans complément d'objet explicite, est plus difficile avec de quelle façon :

[21] Soit dit en passant, je me demande comment vous faites avec ce clavier de machine à écrire allemande (Garat)

[22] * Je me demande de quelle façon vous faites avec ce clavier de machine à écrire allemand.

Enfin l'emploi impossible de comme intégratif met également en évidence l'emploi percontatif interrogatif de comment :

[23] * Je me demande encore comme j'y suis parvenu.

ii) Il est connu que les percontatifs interrogatifs et les intégratifs se distinguent par leurs paraphrases sur le mot en qu- (cf., par exemple Pierrard 1988). C'est ce que montrent ces énoncés avec le pronom humain où seul le pronom intégratif permet d'introduire quelqu'un :

[24] J'aime qui j'aime $\longrightarrow$ j'aime quelqu'un

[25] Je me demande qui viendra $\longrightarrow$ * Je me demande quelqu'un.

Avec une percontative interrogative, il y a une combinaison entre le pronom et la prédication de la subordonnée, d'où la possibilité d'employer le pronom quelque chose $e^{6}$ :

“je me demande quelque chose : l'identification de quelqu'un qui a cette propriété : viendra".

Il s'agit de se positionner, grâce à la matrice, par rapport à l'identification de cette variable. Avec l'intégrative, on ne cherche pas à identifier de variable. Il est difficile de construire un test équivalent pour comment parce qu'il n'existe pas en français de pronom indéfini de la

\footnotetext{
${ }^{6} \mathrm{Cf}$. pour le sémantisme particulier du mot chose qui peut renvoyer à une prédication, Kleiber 1987.
} 
manière. On peut constater néanmoins que les percontatives acceptent plus difficilement que les intégratives l'emploi d'un $\mathrm{GN}^{7}$ :

[26] Je suis comme je suis $\longrightarrow$ Je suis de cette façon ce qui donne pour (15):

[27] * Je me demande encore cette façon / la façon d'y parvenir

[28] Je me demande encore quelque chose

Mais ce test n'est pas complètement fiable. Quelquefois il est possible de construire, à partir d'une percontative interrogative, une paraphrase avec un GN, pourvu qu'il comprenne une relative déterminative :

[29] Je me demande quelle heure il est

[30] Je me demande l'heure qu'il est

même si ce n'est pas possible avec n'importe quel exemple :

[31] Je me demande quel film il va voir

[32] *Je me demande le film qu'il va voir.

Nous verrons donc dans nos exemples si la paraphrase en cette façon ou plus exactement la façon de $V$ ou la façon + relative est possible. Mais nous prendrons les résultats induits par ce test comme une indication qui devra être appuyée par les autres tests, plus fiables.

iii) Une percontative peut se réduire au mot subordonnant ( $«$ sluicing $»^{8}$ ) :

[33] Il fallait que j'avance, même sans savoir vraiment comment, et jusqu'au bout (Roubaud)

ce qui donne pour l'exemple (15):

[34] Je pus me saisir en tâtonnant d'une ancienne paire de lunettes (j'y suis parvenu je me demande encore comment)

Une intégrative ne peut pas aboutir à la même réduction :

[35] Embrassez qui vous voulez $\longrightarrow *$ Embrassez qui

exceptées les intégratives en de quoi qui peuvent accepter d'être réduites au mot en qu- (cf. Lefeuvre 2006b) :

[36] On pleurait beaucoup en ce temps-là, mais quelquefois il y avait de quoi (Chandernagor) $^{9}$

\footnotetext{
${ }^{7}$ Equivalent au SN dans la terminologie de Le Goffic 1993.

${ }^{8}$ Cf. notamment Pierrard 1988, Hadermann 1993, Léonarduzzi 2004. Pour le terme de «sluicing», cf. la grammaire générative et, plus récemment, Krazem 2007.
} 
Ce test ne fonctionne pas dans certains cas, lorsque la percontative n'est pas COD d'un verbe recteur (cf. 1.3), ou bien avec les verbes être, aller, faire :

[37] Je me demande comment il va

[38] * Il va je me demande comment ${ }^{10}$

ou encore lorsque la percontative a pour noyau un infinitif, ce qui correspond à 58 exemples soit $16,5 \%$ des percontatives interrogatives :

[39] je pousse des cris de sauvage pour rire, et là aussi elle m'explique comment faire (Garat)

[40] * faire elle m'explique comment

Comme les intégratives adverbiales ne semblent pas pouvoir comporter de noyau infinitival, contrairement aux intégratives pronominales :

[41] * Il a / * il y a comme faire

[42] Il a à qui parler / Il y a de quoi faire

la présence de l'infinitif avec comment nous semble un signe suffisant pour interpréter la subordonnée comme une percontative interrogative.

Voyons à présent ce qu'il en est pour notre corpus.

\subsection{Corpus}

Nous analyserons en premier lieu comment dans les percontatives interrogatives qui occupent la fonction COD (344 exemples) et ensuite comment dans celles qui assument une autre fonction ( 7 exemples).

\subsubsection{Comment dans les percontatives interrogatives en fonction de COD ( 344 exemples)}

Les percontatives interrogatives assument essentiellement la fonction de complément d'objet : c'est le cas pour $98 \%$ des exemples de percontatives interrogatives. C'est dire l'importance du verbe recteur. Les verbes qui introduisent des percontatives interrogatives en fonction complément d'objet direct se distinguent généralement des verbes qui introduisent des intégratives. Ils évoquent, selon Martin 1985, « un univers de croyance (produit par n'importe quel verbe de demande, de verbe d'énonciation ou de verbe épistémique) » et

\footnotetext{
${ }^{9}$ Pour d'autres tests, notamment sur le rôle des prépositions vis-à-vis du verbe de la subordonnée ou de la principale ou encore le cumul des mots en $q u$ - possible dans les percontatives, cf. les auteurs ci-dessus (note 4) et Lefeuvre 2006a.

${ }^{10}$ Une étude fouillée serait nécessaire pour ces trois verbes. Ce qui semble bloquer dans ces exemples comme en (38), c'est que ces verbes peuvent difficilement s'employer sans complément dans ces configurations.
} 
impliquent « la non explicitation de la valeur de $\mathrm{p}$ [dans la subordonnée] ». D’après Serbat (1985), c'est l'existence d'une donnée problématique pour quiconque qui est déterminante. Le verbe introducteur " [appartient] aux classes larges "savoir" ou "dire" ». Le Goffic (1993) reconnaît également les « verbes de connaissance » et les « verbes déclaratifs » mais il met à part demander, « verbe d'action sur autrui ».

Voyons ce qu'il en est dans notre corpus. Le verbe demander correspond à 56 occurrences sur 351 , soit $16 \%$ c'est-à-dire 1 sur 6 . Nous avons vu en 1.1. que les tests s'appliquaient positivement pour l'exemple (15).

En ce qui concerne les verbes de connaissance (classe du "savoir"), le verbe savoir est le plus récurrent de notre corpus pour régir des percontatives interrogatives : sur 344 exemples, 140 comportent ce verbe, soit $40 \%$. Les énoncés comportant le verbe savoir ou le verbe demander correspondent ainsi à plus de la moitié des énoncés (57\%).

Les tests vus plus haut fonctionnent positivement et montrent qu'il s'agit bien de percontatives interrogatives. Ainsi, pour cet exemple :

[43] Si le Docteur Sachs n'était pas venu me faire un calmant, je ne sais pas comment j'aurais passé la nuit. (Winckler)

cela donne :

[44] Je ne sais pas comment est-ce que / de quelle façon / * comme j'aurais passé la nuit (critère 1)

[45] ? Je ne sais pas la façon dont j'aurais passé la nuit (critère 2)

[46] J'aurais passé la nuit je ne sais pas comment (critère 3)

Relevons que comme $P$ est possible lorsque la subordonnée a pour noyau le verbe être :

[47] Ne vous inquiétez pas : il est chez moi. J'espère que vous n'êtes pas trop malheureuse. Vous savez comment il est ! (Buron)

[48] Vous savez comme il est !

On sait que comme pouvait assumer des emplois interrogatifs et percontatifs interrogatifs au $17^{\mathrm{e}}$ siècle mais aussi dans les siècles ultérieurs :

[49] je ne sais comme elle s'accoutumera à mon absence (Mauriac, Frantext, ex. tiré de Moline soumis ${ }^{11}$ )

\footnotetext{
${ }^{11}$ Les exemples de comme interrogatif, relevés par E. Moline dans Frantext au $20^{\mathrm{e}}$ siècle, sont pour nous des exemples archaïsants, comme le montre, dans les emplois négatifs, la présence du seul adverbe négatif ne (exemple 49). Nous différencierons ces emplois archaïsants des emplois tels que (48), avec savoir et le verbe être dans la percontative interrogative, qui nous paraissent plus courants. Pour une classification de savoir comme, $\mathrm{cf}$. Moline soumis a et $\mathrm{b}$.
} 
Pour ce qui est des verbes déclaratifs (classe de "dire"), le verbe dire revient 14 fois, soit dans moins de $5 \%$ des cas :

[50] On n'a plus entre les doigts qu'une poussière de scènes absurdes et l'on ne peut jamais dire ni comment elles se suivent ni comment tout a commencé. (Forest)

Les tests sont bien opérationnels :

[51] et l'on ne peut jamais dire comment est-ce qu' / de quelle façon / * comme elles se suivent (critère1)

[52] ? et l'on ne peut jamais dire la façon dont elles se suivent (critère 2)

[53] les scènes absurdes se suivent et l'on ne peut jamais dire comment (critère 3)

Les autres verbes se partagent entre ces deux dernières classes, ce qui donne dans notre corpus, pour les verbes de connaissance : apprendre, aviser, choisir, comprendre, concevoir, découvrir, entendre, étudier, examiner, observer, prévoir, regarder, (re)trouver, sentir, voir (voilà) et pour les verbes déclaratifs : décrire, enseigner, expliquer, enseigner, indiquer, montrer, raconter, représenter, se souvenir, se rappeler. Avec certains d'entre eux, il existe des configurations, que nous verrons en 3 , où la distinction entre la lecture intégrative ou percontative interrogative est neutralisée ou bien où comment $P$ est plus proche d'une intégrative que d'une percontative interrogative.

Examinons à présent les exemples où comment $P$ épouse une autre fonction.

\subsubsection{Comment dans les percontatives interrogatives sujet, complément ou régime ${ }^{12}$ de préposition, noyau averbal ( 7 exemples)}

Lorsque comment $\mathrm{P}$ assume une autre fonction que celle de COD, les tests fonctionnent moins bien.

Nous n'avons relevé qu'une seule percontative interrogative en fonction sujet :

[54] Comment était sa remplaçante (il y en eut une nécessairement) m'est entièrement sorti de l'esprit. (Roubaud)

qui répond partiellement aux tests vus en 1.1. Ceux concernant le mot en $q u$-fonctionnent sans difficulté :

[55] Comment est-ce que / De quelle façon / *Comme sa remplaçante était m'est entièrement sorti de l'esprit

Le test du sluicing, à cause de la fonction sujet de la percontative, n'est pas applicable. En 
outre, on peut remplacer comment $P$ par la façon dont $P$ :

[56] La façon dont sa remplaçante était m'est entièrement sorti de l'esprit.

ce qui peut s'expliquer également par la fonction sujet qui accepte sans problème un GN défini, comme la façon dont sa remplaçante était.

Deux exemples se trouvent avec n'importe comment, dans une structure grammaticalisée, par exemple :

[57] le rivesaltes, Cadaquès, les galeries d'art, les moules à l'escabèche au soleil couchant, les bébés couverts de sel et de sueur endormis n'importe comment, l'entrée hallucinée dans Burgos surchauffée, tous les tunnels du Mont-Blanc ventre à terre en enfilade jusqu'à l'Argentario (Billetdoux)

Comment $P$ peut constituer le sujet de importe dans des exemples du type :

[58] Peu importe comment il a fait

où sa position postverbale convient bien à la valeur rhématique qu'il prend alors. La grammaticalisation de n'importe comment explique que les tests ne soient plus applicables :

[59] *N'importe comment / *N'importe de quelle façon ils se sont endormis

Deux autres exemples forment avec la préposition un complément du nom :

[60] Elle voyait tout en mal, argumentait, lançait des ordres de : comment tu feras, iras, reviendras, à quelle heure, et par quel chemin, la bifurcation, le virage, pour exorciser l'accident, le retard, l'empêchement, le malheur. (Garat)

[61] Tu pourrais lui téléphoner, lui dire bonjour, lui poser des questions sur ce qu'il fait, comment vont ses enfants, sa femme, si son travail lui plaît. (Mrejen)

Avec les verbes faire et aller, la paraphrase avec la façon dont $P$ est difficile à appliquer, comme c'est le cas avec de quelle façon. Un autre verbe rendrait ces tests positifs :

[62] Tu pourrais [...] lui poser des questions sur ce qu'il fait, comment il a monté son affaire

[63] Tu pourrais [...] lui poser des questions sur ce qu'il fait, la façon dont il a monté son affaire.

\footnotetext{
${ }^{12}$ Pour cette terminologie, cf. Le Goffic 1993.
} 
Avec de quelle façon, la présence des prépositions introduisant la percontative rend la paraphrase malaisée, quel que soit le verbe de la subordonnée :

[64] ? Tu pourrais lui téléphoner, lui dire bonjour, lui poser des questions sur ce qu'il fait, de quelle façon il a monté son affaire.

Le test des autres mots en $q u$ - fonctionne positivement :

[65] Elle voyait tout en mal, argumentait, lançait des ordres de : comment est-ce que tu feras, iras, reviendras / *comme tu feras

[66] Tu pourrais lui téléphoner, lui dire bonjour, lui poser des questions sur ce qu'il fait, comment est-ce que ses enfants vont $/ *^{*}$ comme ses enfants vont

Le test du sluicing est peu aisé à appliquer à cause de la fonction de la percontative et des verbes aller et faire :

[67] *Ses enfants vont il lui pose des questions sur comment

[68] *Tu feras elle lance des ordres de comment

Seul le test des mots en qu- en est-ce que et comme permet de classer ces propositions dans les percontatives interrogatives. La présence de $s i P$ en (61) va dans ce sens.

Comment $P$ peut enfin constituer le noyau d'un titre (2 exemples), parfois en coordination ou juxtaposition avec un GN :

[69] [...] différentes sections [...] me séduisent : « L'amour des hommes varie autant que son objet »; «Comment l'amour tyrannise »; « Causes de la mélancolie amoureuse : le regard, la beauté du visage, des yeux, d'autres parties du corps »; « Symptômes »; « Signes pronostiques »; (Pontalis)

Il est possible de remplacer comment $P$ par la façon dont $P$ puisque les titres se construisent sans problème avec un GN :

«La façon dont l'amour tyrannise »

Le test de la réduction au mot en $q u$ - ne peut pas être appliqué en l'absence de verbe recteur. Les tests des mots en $q u$ - fonctionnent positivement :

[70] [...] différentes sections [...] me séduisent : « L'amour des hommes varie autant que son objet »; «Comment est-ce que / De quelle façon l'amour tyrannise »

Avec comme:

[71] Comme l'amour tyrannise

le sens est celui de la manière remarquable. Il s'agit d'un emploi exclamatif. Ce n'est pas un emploi intégratif. En outre, la proposition en comment $P$ peut alors comprendre un infinitif : 
[72] ils embarquèrent dans le désordre fauteuils, sofas, [...], essoreuse à salade et livres pour se divertir, L'Art de plier des serviettes pour décorer la table, Comment lire son avenir dans les cartes ou Les 1000 astuces pour se simplifier la vie, sans oublier quelques ratons laveurs. (Flem)

Tous ces points permettent de voir dans cette structure une percontative interrogative. Il est inutile de parler d'emploi elliptique, quelle que soit la configuration où elle se situe. Equivalent d'un GN, la subordonnée en comment $P$ suit le même rôle qu'un GN dans cette position : dans la configuration habituelle d'un titre, elle constitue un noyau averbal. Ici, ce titre est dans la dépendance argumentale de embarquèrent, en (72), en tant que COD, et figure comme élément d'une énumération en (69).

Qu'en est-il de comment percontatif exclamatif?

\section{Comment percontatif exclamatif ( 3 exemples)}

L'emploi exclamatif de comment semble bien attestée jusqu'au $18^{\mathrm{e}}$ siècle, que ce soit en emploi indépendant (73) ou en subordonnée (74) :

[73] Avec quelle finesse il sçavoit attaquer ma pudeur, et me la faire oublier ! Combien il paroît le desir, et comment il le faisoit naître ! (Crébillon fils)

[74] Vous pouvez vous figurer comment je suis demeurée interdite en ouvrant votre lettre, qui m'apprend la première nouvelle... grand dieu du ciel et de la terre ! (Prévost l'Abbé)

Les grammaires reconnaissent ce type d'emploi à comme mais pas à comment (cf. Moline 2009). On reconnaît pourtant ici le sémantisme de la «manière remarquable » due à quelque chose d'inhabituel ou d'inattendu (cf. Fuchs \& Le Goffic, 2005 et Moline soumis b). Au $20^{\mathrm{e}}$ siècle, on trouve des exemples de comment exclamatif assorti d'une proposition en que $P$, en emploi indépendant :

[75] Ah ! la la ! s'il n'y avait pas le jazz, comment que je mettrais les voiles ! (Fallet, 1947)

ou subordonnant :

[76] T'as vu comment que c'était petit !... (Céline, 1932)

E. Moline (2009) signale la possibilité pour comment d' assurer, en français contemporain, un emploi d'exclamatif sans que:

[77] Comment il parle, celui-là !

Un seul exemple de notre corpus se rapproche de ces énoncés, sans que : 
[78] Abominable comme une histoire d'amour ne peut pas se liquider, comment froide elle peut encore brûler, brûler horriblement. (Guibert)

Cet énoncé comporte à la fois comment $P$ et comme $P$ en tant qu' «exclamatives indirectes introduites par un adjectif» (cf. Bacha 2000 ; et Moline 2009). On voit donc que la répartition entre comment percontatif interrogatif et comme percontatif exclamatif n'est pas tout à fait effective.

Cela dit, il nous semble que comme assure plus facilement cet emploi d'exclamatif que comment. En effet la valeur de haut degré n'est pas toujours nette avec comment. C'est le cas de ces deux exemples :

[79] c'était beau, c'était juste, je buvais tes paroles, j'entendais la musique des voix, le duo, le chœur que tu étais à toi toute seule et comment tu savais manier le motif des enfances en sourdine, le fouet du coup d'archet, plaquer l'accord. (Garat)

[80] Alors sa grand-mère Léonie apprend comment son fils la délaisse, elle la fait venir à la campagne chez elle, elle la garde deux années. (Garat)

En (79) et (80), comment ne répond pas aux tests des percontatives interrogatives :

[81] J'entendais [...] le chœur que tu étais à toi toute seule et * comment est-ce que /* de quelle façon tu savais manier le motif des enfances en sourdine

[82] Alors sa grand-mère Léonie apprend * comment est-ce que / * de quelle façon son fils la délaisse.

La réduction au mot en $q u$ - :

[83] * Tu savais manier le motif des enfances en sourdine j'entendais comment

[84] * Son fils la délaisse, sa grand-mère Léonie apprend comment.

est malaisée ainsi que la paraphrase par la façon dont (ce qui permet de supposer qu'il ne s'agit pas d'un emploi intégratif) :

[85] * J'entendais [...] le chœur que tu étais à toi toute seule et la façon dont tu savais manier le motif des enfances en sourdine

[86] * Alors sa grand-mère Léonie apprend la façon dont son fils la délaisse.

Enfin, comme exclamatif (et non intégratif) ou à quel point peuvent paraphraser comment :

[87] J'entendais [...] le chœur que tu étais à toi toute seule et comme / à quel point tu savais manier le motif des enfances en sourdine, le fouet du coup d'archet, plaquer l'accord.

[88] Alors sa grand-mère Léonie apprend comme / à quel point son fils la délaisse.

Ces tests montrent que (79) et (80) ne sont pas intégratifs : comme, mis à la place de comment, prend une valeur exclamative et la paraphrase en la façon dont $P$ est impossible. Nous avons 
étiqueté ces exemples comme exclamatifs mais ils posent un problème de classement. En effet, la lecture induite en (87) et (88) semble assez différente d'un point de vue sémantique : le degré parait surajouté. Cela peut s'expliquer parce que délaisser et manier prennent bien des circonstants de manière mais moins facilement des adverbes portant sur le degré, contrairement à un verbe tel que souffrir par exemple. Ces exemples ne sont donc pas nettement exclamatifs. Appartiennent-ils alors à une autre catégorie, qualifiée de « phrase à interprétation indéfinie » dans Moline soumis b ? Nous pouvons considérer aussi que c'est un problème inhérent à tout essai de catégorisation: ces énoncés ne représentent pas des exemples prototypiques mais des exemples qui ont des «airs de ressemblance » avec la catégorie des exclamatifs tout en se distinguant des prototypes (cf. Kleiber 1990).

Comme assure plus facilement cet emploi d'exclamatif que comment. Dans quelques exemples (3) de notre corpus :

[89] Jubilation d'Hitler quand il découvre en 1919 dans quelque brasserie munichoise comment la parole peut fasciner un auditoire : «Ce dont j'avais toujours eu la prescience se trouvait confirmé : je savais parler. » (Pontalis)

le remplacement de comment par comme :

[90] Jubilation d'Hitler quand il découvre en 1919 dans quelque brasserie munichoise comme la parole peut fasciner un auditoire

entraîne nettement une interprétation exclamative alors qu'avec comment, l'interprétation peut rester interrogative, comme le montre l'application positive aux tests :

[91] Jubilation d'Hitler quand il découvre en 1919 dans quelque brasserie munichoise comment est-ce que / de quelle façon la parole peut fasciner un auditoire

[92] La parole peut fasciner un auditoire il découvre comment.

Pour cette raison, nous avons classé ces exemples comme interrogatifs.

Voyons à présent si comment peut assurer un rôle d'intégratif.

3. Comment intégratif (20 exemples de comment intégratif +20 exemples de comment intégratif / percontatif interrogatif)

Après avoir vu que comment a toujours assuré des emplois d'intégratif, de l'ancien français au français contemporain, nous examinerons deux emplois plus délicats à catégoriser : i) comment $P$ régi par des verbes pouvant introduire à la fois des percontatives en comment $P$ et des intégratives en comme $P$ et ii) comment $P$ constituant un noyau averbal. 


\subsection{Comment $P$ régi par un verbe introducteur d'intégratives ( 5 exemples)}

Un bref rappel historique nous permettra d'éclairer quelques emplois du français contemporain. En ancien français jusqu'au $16^{\mathrm{e}}$ siècle, comment a pu être relatif avec un antécédent nominal. Il pouvait effectivement anaphoriser des noms tels que façon et manière :

[93] Comment Pantagruel feut malade, et la façon comment il guerit. (Rabelais)

[94] Et le duc luy donna sa robe, laquelle luy avoit promise et luy failloit compter au duc la maniere comment avoit obtenu le cheval. (Anonyme, Ulenspiegel, 1530)

Gougenheim signale que comme connaît aussi cet emploi de relatif (1984 : 93), avec le même type de mots comme antécédents (cf. aussi Pierrard 1998) :

[95] Ils accorderent ensemble le moyen comme ilz se pourroient veoir hors de la veue des autres (Marguerite de Navarre, ex. tiré de Gougenheim 1984)

$\mathrm{Au} 17^{\mathrm{e}}$ siècle, ces emplois semblent avoir disparu. Comment et comme ont été remplacés par dont :

[96] compter au duc la maniere dont il avait obtenu le cheval.

En outre, comment a pu connaître des emplois d'intégratif, là où l'on attendrait aujourd'hui plutôt comme :

[97] Et, à la porte du moustier, l'evesque du Don les print par les mains et les espousa comment il les avoit fianciez (Jouvencel, ex. tiré de Martin et Wilmet 1980).

Il était possible de construire une intégrative en comment avec le verbe être :

[98] « Touttesfois, comme j'ay dit, le dangier est comment on pourra faire de cest enfant baitairt (Vigneulles, 1515)

Aujourd'hui, comment connaît toujours des emplois intégratifs (3 exemples), en fonction d'attribut :

[99] Une des figures des rituels établis, qui les montre conduisant en parallèle, c'est comment ils ramènent les voitures neuves de l'usine (Bon)

ou de $\operatorname{COD}^{13}$ :

[100] J'aime comment T. m'étreint (Guibert)

[101] J'aime l'imprévisibilité du blue-jean : comment, après lavage, il rétrécit, vieillit, pâlit. (Lève)

Les verbes aimer et être régissent des intégratives mais pas des percontatives interrogatives :

[102] J'aime * comment est-ce qu' / * de quelle façon il / comme T. m'étreint

\footnotetext{
${ }^{13}$ Pour l'exemple 101, à cause du deux-points, on pourrait proposer une autre fonction qui rapprocherait la subordonnée d'un noyau averbal (cf. 3.3).
} 
[103] C'est * comment est-ce qu' / * de quelle façon / comme ils ramènent les voitures neuves de l'usine

Comment, dans les exemples, se paraphrase alors par la façon dont :

[104] J'aime la façon dont il m'étreint

[105] C'est la façon dont ils ramènent les voitures neuves de l'usine Il ne peut pas constituer à lui seul la subordonnée :

[106] * Il m'étreint j'aime comment

[107] * Ils ramènent les voitures neuves de l'usine c'est comment.

En tant qu'intégratif, comment accepte d'être régi par très peu de verbes. Cela semble possible avec le champ lexical de aimer: apprécier, détester, haïr, estimer. Sans doute comment apparaît-il dans ces exemples parce que comme offre avec certains verbes (souffrir : J'aime comme Pierre souffre) une double interprétation : lecteur intégrative ou lecture exclamative, paraphrasable par à quel point. Dans ces exemples, notamment celui avec être, la valeur de la manière ressort davantage avec comment qu'avec comme ${ }^{14}$.

Deux autres exemples de comment $P$ constituent, avec la préposition, un COI (108) et un circonstant (109)

[108] En entendant Elio, ainsi, allongée près du corps nu de Ginetta, j'ai pensé à leur amour, non pas à cet amour dans le temps, mais dans l'espace, comment cet amour d'Elio s'était enraciné et se nourrissait chaque jour de cette femme. (Duras)

[109] Vous savez, j'ai beaucoup lu sur le sujet... comment les maladies se propageaient tant qu'on faisait ça n'importe où (Winckler)

Ces propositions surviennent dans un paradigme de GN, avec des verbes qui ne régissent pas de percontatives (penser à, lire) mais qui acceptent des intégratives :

[110] Je pense à qui je pense !/ Je lis ce que je lis !

[111] * Je pense à quel film je verrai / * Je lis sur quel livre je veux

Dans les deux cas, la présence du GN facilite celle de comment $P$ :

[112] ? J'ai pensé à comment cet amour d'Elio s'était enraciné

[113] ? J'ai beaucoup lu sur comment les maladies se propageaient

Nous verrons donc en ce comment un intégratif, comme le montre l'application aux tests vus en 1 , avec les mots en $q u$ - :

[114] J'ai pensé à leur amour, [...], * comment est-ce que / * de quelle façon / comme cet amour d'Elio s'était enraciné 
[115] Vous savez, j'ai beaucoup lu sur le sujet... * comment est-ce que / * de quelle façon / comme les maladies se propageaient

Comment est paraphrasable par la façon dont :

[116] J'ai pensé à leur amour, [...], à la façon dont cet amour d'Elio s'était enraciné et se nourrissait chaque jour de cette femme.

[117] ... Vous savez, j'ai beaucoup lu sur le sujet... sur la façon dont les maladies se propageaient

Il ne peut pas constituer à lui seul la subordonnée :

[118] * Cet amour d'Elio s'était enraciné j'ai pensé comment

[119] * Les maladies se propageaient j'ai beaucoup lu comment

pour une double raison : comment n'est pas percontatif et il complète une préposition (cf. 1.3). La subordonnée en comme $P$ autorise ici une double interprétation : lecture intégrative où comme prend la valeur de la manière et lecture exclamative où comme se glose alors par $\grave{a}$ quel point :

[120] J'ai pensé à leur amour, non pas à cet amour dans le temps, mais dans l'espace, comme / à quel point cet amour d'Elio s'était enraciné et se nourrissait chaque jour de cette femme

[121] ... Vous savez, j'ai beaucoup lu sur le sujet... comme / à quel point les maladies se propageaient.

Comment paraît plus ancré sur la valeur de la manière.

\subsection{Comment $P$ régi par un verbe introducteur de percontatives interrogatives ou} d'intégratives ( 1 exemple d'intégrative et 20 exemples de percontatives interrogatives / intégratives)

Comment $P$ peut se trouver régi par des verbes qui peuvent introduire des percontatives interrogatives ou des intégratives. Avec certains des verbes signalés en 1, les tests ne permettent pas toujours de délimiter clairement le type de subordonnée. C'est le cas du verbe voir (voici/voilà), de regarder et de raconter qui tous trois acceptent des subordonnées en comment $P$ ou comme $P: 21$ exemples de notre corpus acceptent le

\footnotetext{
${ }^{14} \mathrm{Cf}$. Moline 2009 qui rappelle l'étymologie de comment, doublement marqué pour la valeur de la manière (avec com et -ment), contrairement à comme.
} 
remplacement de comment $P$ par comme $P$ intégratif (et non exclamatif) ${ }^{15}$. Ainsi en est-il de cet exemple avec la forme voilà :

[122] Et pour tromper son monde, voilà comment il a procédé : alors qu'il n'était question que des élections, il a détourné la question (Duras)

[123] Voilà comme il a procédé.

La paraphrase avec la façon dont est également possible :

[124] Voilà la façon dont il a procédé

ainsi que la réduction au seul adverbe comment :

[125] Et pour tromper son monde, il a procédé voilà comment.

ou encore la paraphrase par comment est-ce que, de quelle façon :

[126] Voilà comment est-ce qu' / de quelle façon il a procédé

On peut faire l'hypothèse que la distinction d'emplois entre les emplois percontatifs et les emplois intégratifs est ici neutralisée. Le Goffic (1987) signale ainsi que pour certains verbes, il est parfois artificiel de trancher entre les deux interprétations, notamment en ce qui concerne les mots en qu- qui renvoient à de l'inanimé. Ces cas de figure ne se borneraient donc pas à ce sémantisme.

Dans un exemple de notre corpus, seule la lecture intégrative est possible :

[127] Ils racontaient l'histoire de Râma, son exil dans la forêt, et comment Sîtâ fut enlevée par le démon Râvana et qu'il fallut à Râma livrer, aux côtés du dieu-singe Hanuman, la plus grande bataille de tous les temps (Forest)

La proposition en comment $P$, régie par le verbe raconter, est coordonnée avec un GN à sa gauche et avec une complétive à sa droite. Le test de réduction au seul mot en qu- est difficile à appliquer à cause des autres compléments de racontaient. Seuls les tests propres à l'emploi intégratif fonctionnent positivement :

[128] Ils racontaient l'histoire de Râma, son exil dans la forêt, et * comment est-ce que / * de quelle façon / comme Sîtâ fut enlevée par le démon Râvana et qu'il fallut à Râma livrer, [...], la plus grande bataille de tous les temps

[129] Ils racontaient l'histoire de Râma, son exil dans la forêt, et la façon dont Sîtâ fut enlevée par le démon Râvana et qu'il fallut à Râma livrer, [...], la plus grande bataille de tous les temps.

\footnotetext{
${ }^{15}$ Nous nous basons aussi sur une recherche d'après Frantext (de 1950 à nos jours) et internet. Avec les verbes suivants, comprendre, décrire, montrer, représenter, se rappeler, se souvenir, nous n'avons trouvé aucun exemple avec comme intégratif.
} 
Ici c'est la présence de la complétive qui bloque la lecture percontative. En la supprimant, les paraphrases en comment est-ce que et de quelle façon sont de nouveau possibles :

[130] Ils racontaient l'histoire de Râma, son exil dans la forêt, et comment est-ce que / de quelle façon Sîtâ fut enlevée par le démon Râvana.

En effet la coordination entre une percontative et une complétive semble impossible :

[131] * Je sais comment ton père a fait et que ta mère est venue.

Cela dit, il existe des emplois de voir (voici / voilà), se regarder et de raconter qui rejettent les subordonnées en comme, ce qui permet de considérer ces dernières comme des percontatives interrogatives :

[132] Il disait juste qu'il n'avait aucune oreille dans la maison en raison de l'état actuel de la littérature et qu'il ne voyait pas comment il défendrait un manuscrit pareil devant le Comité (Garat)

[133] * Il ne voyait pas comme il défendrait un manuscrit pareil.

Peut-être est-ce ici la présence de la négation qui oriente l'exemple (132) vers une valeur percontative. D'autres verbes tels que comprendre, décrire, montrer, se rappeler, représenter, se souvenir acceptent la paraphrase avec la façon dont mais pas avec comme $P$ intégratif $^{16}$ :

[134] Je commence un peu à comprendre ce virus, comment il se déplace. (Guibert)

[135] Je commence un peu à comprendre ce virus, comment est-ce qu' / de quelle façon /* comme il se déplace.

[136] Je commence un peu à comprendre ce virus, la façon dont il se déplace La réduction du mot en $q u$ - est également possible :

[137] Ce virus se déplace je commence un peu à comprendre comment Nous considérerons donc ces propositions comme des percontatives interrogatives.

\subsection{Comment $P$ comme noyau averbal (14 exemples)}

Cette configuration regroupe 14 exemples tirés d'un même ouvrage où comment $P$ est inséré dans un texte et ne constitue pas un titre. Il peut être coordonné à un GN, comme ici :

[138] lorsque nous sommes par exemple ensemble dans un sauna : l'impression d'être "hors-jeu", déplacé, mutilé... Le Noir dans la salle à manger : ses gestes surraffinés, et comment il se met à tourner sa cuillère dans sa tasse de café, en rond, interminablement, les yeux dans le vide (Guibert)

\footnotetext{
${ }^{16}$ Parfois ils acceptent la paraphrase avec comme $P$ percontatif exclamatif.
} 
Comment il se met à tourner sa cuillère dans sa tasse de café [...] est sur le même plan syntaxique que ses gestes sur-raffinés, aisément glosable par la façon dont ou comme P :

[139] Le Noir dans la salle à manger : ses gestes sur-raffinés, et la façon dont il se met à tourner sa cuillère dans sa tasse de café.

[140] Le Noir dans la salle à manger : ses gestes sur-raffinés, et comme il se met à tourner sa cuillère dans sa tasse de café.

Contrairement aux propositions qui surviennent dans les titres, les tests propres aux emplois percontatifs sont ici négatifs :

[141] Le Noir dans la salle à manger : ses gestes sur-raffinés, et * comment est-ce qu' / * de quelle façon il se met à tourner sa cuillère dans sa tasse de café

Le test de réduction au seul mot en $q u$-, en l'absence de verbe, est inapplicable. Il s'agit donc d'un emploi intégratif.

En ce qui concerne la fonction, le GN ses gestes sur-raffinés et la subordonnée en comment pourraient être interprétés comme des prédicats assertant l'existence de telle ou telle propriété :

"En ce qui concerne l'existence du Noir dans la salle à manger, il y a ses gestes surraffinés et la façon dont il se met à tourner sa cuillère".

ou bien comme des prédicats seconds, non assertés. Mais le fait que ce soit des prédicats n'est pas évident, à cause notamment de l'absence de tout « marqueur de prédication » (Lefeuvre 1999). Il est impossible d'ailleurs de faire précéder comment $P$ d'un tel marqueur :

[142] ... Le Noir dans la salle à manger : toujours ses gestes sur-raffinés, et * toujours comment il se met à tourner sa cuillère dans sa tasse de café

Dans ces exemples, comment $P$ ne peut pas être complément de il y $a$ :

[143] * Il y a comment P

ce qui peut expliquer que nous ayons une structure syntaxique averbale. On retrouve régulièrement le schéma suivant : un GN défini, support thématique, est suivi, après un deux points, de GN donnant telle ou telle caractéristique.

Ce n'est pas le cas de l'exemple suivant :

[144] Très beau chez Chostakovitch : comment on a l'impression que le musicien se souvient de la façon dont il percevait la musique quand il était enfant. (Guibert)

Le fait que comment $P$ forme un prédicat semble plus net dans cet exemple. C'est évident pour le groupe adjectival (très beau chez Chostakovitch). Cet exemple rappelle des configurations averbales telles que (cf. Lefeuvre 2007) : 
[145] Dernier enseignement de ce sondage, la volonté européenne de voir prévaloir la diplomatie dans l'affaire de l'uranium iranien (Le Monde)

paraphrasable par :

“Il y a un dernier enseignement de ce sondage, c'est la volonté européenne de voir prévaloir la diplomatie dans l'affaire de l'uranium iranien".

Il pourrait pareillement se paraphraser de la façon suivante :

"Il y a quelque chose de très beau chez Chostakovitch : c'est comment on a l'impression que le musicien se souvient de la façon dont il percevait la musique quand il était enfant".

\section{Comment complétif ? (7 exemples)}

Le sens de comment, dans deux types de cas, porte sur la validité de la subordonnée. Nous parlerons alors de «sens énonciatif» dans la mesure où l'adverbe porte alors sur la phrase dans son ensemble et plus précisément sur la relation entre P et son énonciation. Il se rapproche alors du que complétif.

Tout d'abord, des subordonnées en comment (2), introduites par un verbe déclaratif, comportent des circonstants de manière :

[146] Il nous expliqua un jour sur quelques exemples comment, d'un tout petit carton de papier étroit, d'une fiche couverte de son écriture nette mais minuscule, il pouvait sans hésitation et rapidement extraire le contenu factuel (Roubaud)

[147] je découvrais au fil des pages leur transposition dans la réalité et comment, avec les moyens du bord et au gré des circonstances qui étaient à portée de sa main, elle avait miraculeusement adapté le roman de Virginia Woolf (Bouillier)

La présence de compléments de manière - d'un tout petit carton de papier étroit, d'une fiche couverte de son écriture nette mais minuscule / avec les moyens du bord et au gré des circonstances qui étaient à portée de sa main - déplace le sens de comment sur un sens énonciatif qui pourrait se gloser par :

[148] Il nous expliqua un jour sur quelques exemples comment il était possible que, d'un tout petit carton de papier étroit, d'une fiche couverte de son écriture nette mais minuscule, il pouvait sans hésitation et rapidement extraire le contenu factuel.

[149] je découvrais au fil des pages leur transposition dans la réalité et comment il était possible que, avec les moyens du bord et au gré des circonstances qui étaient 
à portée de sa main, elle avait miraculeusement adapté le roman de Virginia Woolf.

Ce type d'emploi a déjà été signalé par quelques auteurs comme Sandfeld (1965) et Wartburg \& Zumthor (1989) pour des exemples tels que :

[150] Je ne comprends pas comment tu épouses une femme qui a deux enfants (exemple tiré de Sandfeld 1977)

Cela rappelle un emploi de comment dans les interrogatives ${ }^{17}$ :

[151] À votre vue elles se lèvent comme des ressorts et se jettent dans vos bras en criant :

- Mamie !... Madame !... On n'a rien fait !

- Comment ça, vous n'avez rien fait ? s'exclame un énorme gorille à côté d'elles, et mon pouce alors?

Il agite sous votre nez le doigt en question empaqueté dans un gros pansement (Buron)

Comment ça permet de remettre en question la validité de l'énoncé précédent : on n'a rien fait.

Le deuxième cas de figure correspond à des subordonnées (3) dont le verbe se combine mal avec un circonstant de manière :

[152] Je pense aussitôt au merveilleux presque centenaire André Spire, un tout petit vieillard aux yeux jeunes, que j'entendis un jour raconter comment, ne tenant plus à la vie que par un fil ténu, incertain, il passait chaque matin une heure hors, audessus de lui-même, une fois réveillé, à 'rentrer dans son corps' (Roubaud, Poésie : récit, 2000)

[153] Alors, je découvre comment c'est vous qui m'apprenez l'amour des corps. (Billetdoux)

[154] J'ai proposé cet itinéraire exprès, mais pas pour les raisons que vous imaginez. L'aller et retour sibérien me permettait de démontrer comment on pouvait enchaîner plusieurs étapes dans un ensemble à grande échelle. (Mathews)

De telles configurations verbales - passer chaque matin une heure à, c'est quelqu'un qui, enchaîner plusieurs étapes — admettent difficilement un complément de manière. La seule possibilité de comprendre ces énoncés est d'introduire un sens énonciatif :

\footnotetext{
${ }^{17}$ Voir aussi Wilmet 2003 pour cet emploi de comment (« complément transprédicationnel de l'énonciation ») : Comment Pierre a-t-il réussi ? qui peut se comprendre de la façon suivante : "on se demande par quel miracle il a réussi”.
} 
[155] J[e l']entendis un jour raconter comment il était possible qu'il passât chaque matin une heure hors $[\ldots]$

[156] Alors, je découvre comment il est possible que ce soit vous qui m'appreniez l'amour des corps.

[157] L'aller et retour sibérien me permettait de démontrer comment il était possible qu'on pût enchaîner plusieurs étapes dans un ensemble à grande échelle.

Nous avons trouvé deux autres exemples où le verbe de la matrice régissait une subordonnée en comment $P$ et une subordonnée en comme $P$ :

[158] car je sentais comme cette petite bête se faisait lourde sur moi, elle qui ne pesait presque rien, par peur sans doute qu'on ne l'arrache à cette sécurité toute neuve, comment, par d'infimes pressions, elle appuyait son nez, son petit mufle, sur ma peau. (Dupérey)

[159] La naissance d'un amour (la littérature allemande) : me souvenir comment enfant j'avais déployé dans ma chambre la carte géographique de l'Allemagne, et comme j'aimais cette forme comme j'aurais pu aimer un corps (Guibert)

On retrouve les mêmes problèmes que précédemment: déployer n'a aucune affinité particulière avec un complément de manière et l'énoncé (158) comporte déjà un complément de manière — par d'infimes pressions.

Dans tous ces énoncés, les tests de reconnaissance des percontatives fonctionnent moins bien :

[160] Il nous expliqua un jour sur quelques exemples ? comment est-ce que / ? de quelle façon, d'un tout petit carton de papier étroit, d'une fiche couverte de son écriture nette mais minuscule, il pouvait sans hésitation et rapidement extraire le contenu factuel

La réduction au seul mot en $q u$ - paraît difficile :

[161] * D'un tout petit carton de papier étroit, d'une fiche couverte de son écriture nette mais minuscule, il pouvait sans hésitation et rapidement extraire le contenu factuel, il nous expliqua comment.

Il est également malaisé d'employer le verbe demander, par excellence introducteur des percontatives interrogatives :

[162] ? Il nous demanda un jour sur quelques exemples comment, d'un tout petit carton de papier étroit, d'une fiche couverte de son écriture nette mais minuscule, on pouvait sans hésitation et rapidement extraire le contenu factuel 
Tout cela peut s'expliquer parce qu'il est difficile d'attribuer à comment son sens habituel de manière.

Le sens énonciatif que prend alors comment rapproche ce type de subordonnées des complétives, ce qui donne pour (146) :

[163] Il nous expliqua un jour sur quelques exemples que, d'un tout petit carton de papier étroit, d'une fiche couverte de son écriture nette mais minuscule, il pouvait sans hésitation et rapidement extraire le contenu factuel.

Une complétive peut alors être coordonnée à comment $P$, contrairement à (131) :

[164] Il nous expliqua un jour sur quelques exemples comment, d'un tout petit carton de papier étroit, d'une fiche couverte de son écriture nette mais minuscule, il pouvait sans hésitation et rapidement extraire le contenu factuel, et que pour cette raison il recommandait ce procédé.

Dans les deux cas, le mot en $q u$ - n'assume pas de fonction intra-prédicative. La différence, c'est que que est un « groupe pronominal attribut d'un être sous-jacent » (Le Goffic 1993) :

"Que (ce que) P est"

alors que comment est un circonstant extra-prédicatif. En outre, contrairement au complétif que, comment permet, dans tous ces exemples, d'introduire un commentaire et de relier un fait à la façon de le dire, ce qui donne par exemple pour (147) :

[165] Comme il nous l'expliqua, d'un tout petit carton de papier étroit, d'une fiche couverte de son écriture nette mais minuscule, il pouvait sans hésitation et rapidement extraire le contenu factuel.

Comment signale les conditions d'énonciation dans lesquelles l'action de la subordonnée P est valide.

Nous avons déjà relevé un cheminement similaire avec comme quoi $P$, proche du sens d'une complétive :

[166] François risqua le tout pour le tout. Il raconta au vieux comme quoi Guillaume Gourvennec désirait le voir mourir le plus vite possible, et, d'abord, ne plus le garder chez lui. (Queffelec).

Rappelons que, en moyen français, comment pouvait assurer un emploi proche de comme quoi en complétant un nom (cf. Martin et Wilmet 1980) :

[167] Faictes unes lettres comment je fais mon filz le Jouvencel regent du royaume d'Amydoine (Jouvencel, ex. tiré de Martin et Wilmet 1980) 
De fait il sera relayé à partir du $17^{\mathrm{e}}$ siècle par comme quoi (cf. Lefeuvre 2006a). On peut faire dès lors l'hypothèse que ce type de possibilité d'emploi est propre à des subordonnants de manière.

Le tableau suivant récapitule les différents emplois de comment avec les données chiffrées liées à notre corpus.

\begin{tabular}{|l|l|l|l|l|l|}
\hline Exemples & $\begin{array}{l}\text { Comment } \\
\text { percontatif } \\
\text { interrogatif }\end{array}$ & $\begin{array}{l}\text { Comment } \\
\text { percontatif } \\
\text { exclamatif }\end{array}$ & $\begin{array}{l}\text { Comment } \\
\text { percontatif } \\
\text { interrogatif/ } \\
\text { intégratif }\end{array}$ & $\begin{array}{l}\text { Comment } \\
\text { intégratif }\end{array}$ & $\begin{array}{l}\text { Comment } \\
\text { proche du } \\
\text { complétif }\end{array}$ \\
\hline 401 & 351 & 3 & 20 & 20 & 7 \\
\hline $100 \%$ & $87,75 \%$ & $0,75 \%$ & $5 \%$ & $5 \%$ & $1,75 \%$ \\
\hline
\end{tabular}

\section{Conclusion}

Ainsi les grandes lignes d'emplois de comment sont nettes : dans une grande majorité $(87,75 \%)$, les énoncés comportant comment relèvent d'un emploi percontatif interrogatif. Cela dit il apparaît que comment subordonnant peut bel et bien assurer, même si c'est dans une fréquence moindre, d'autres types d'emplois : percontatif exclamatif, intégratif et enfin un emploi proche d'un complétif. La possibilité pour comment d'apparaître dans des emplois percontatifs exclamatifs ou intégratifs perturbe l'efficacité du test en comment et comme chargé d'aider à catégoriser les cas difficiles, notamment dans les exemples où les verbes recteurs sont voir (voici / voilà), regarder ou raconter. Nous avons vu également que dans plusieurs cas de figure, la catégorisation de comment s'avère difficile : plutôt que de multiplier les catégories, nous nous en sommes tenue au cadre conceptuel issu de Le Goffic 1993, 2002, qui permet de classer la grande majorité des exemples. Mais ce faisant, nous adoptons, plutôt que la vision aristotélicienne de la catégorisation, une vision plus souple qui tienne mieux compte de la complexité du réel et qui est exposée notamment dans la Sémantique du prototype de Kleiber 1990 : les catégories définies se caractérisent par des prototypes mais également par des éléments qui connaissent des airs de ressemblance avec ces prototypes tout en s'en distinguant.

\section{Bibliographie}


BACHA J., 2000, L'exclamation. Approche syntaxique et sémantique d'une modalité énonciative, Paris, L’Harmattan.

FOURNIER N., 1998, Grammaire du français classique, Paris, Belin.

Fuchs C. \& Le Goffic P. (2005), «La polysémie de comme», in O. Soutet (ed) La Polysémie, Paris, PUPS : 267-291.

Gougenheim G., 1984, Grammaire de la langue française du seizième siècle, Paris, Picard.

Grevisse M., 1988, Le Bon Usage, Paris, Duculot.

Hadermann P., 1993, Etude morphosyntaxique du mot où, Paris-Louvain-la-Neuve, Duculot.

HiRSCHBÜHLER P., 1987, "French quoi and its clitic allomorph que », Studies in romance Language (Neidle C. et Nunez Cedeno R.A.), Dordrecht, Foris publications.

KLEIBER G., 1987, « Mais à quoi sert donc le mot CHOSE ? Une situation paradoxale », Langue française, $\mathrm{n}^{\circ} 73$, p. 109-127.

KLEIBER Georges, 1990, La Sémantique du Prototype, Paris, PUF

KRAZEM M., 2007, «Quand le chevillage percontatif questionne les interrogatives indirectes », in Charolles M., Fournier N., Fuchs C. \& Lefeuvre F. (eds), Parcours de la phrase, Mélanges en l'honneur de Pierre Le Goffic, Paris, Ophrys, p. 13-28.

LEARD J.-M., 1992, Les gallicismes, Etude syntaxique et sémantique, Paris-Louvain-laNeuve, Duculot.

LE GofFIC P., 1987, «Sur l'ambiguïté des relatives / interrogatives indirectes en ce qui, ce que », in Fuchs C. (ed), L'ambiguïté et la paraphrase : opérations linguistiques, processus cognitifs, traitements automatisés, Centre de publication de l'Université de Caen, p. 83-88

LE GofFIC P., 1993, Grammaire de la phrase française, Paris, Hachette.

LE GofFIC P. (ed.), 2002, Interrogation, indéfinition, subordination, Verbum, $\mathrm{n}^{\circ} \mathrm{XXIV}, 4$.

LE GofFIC P. (ed.), 2007, Les mots en qu-du français, Lexique, $n^{\circ} 18$.

LEFEUVRE F., 1999, La phrase averbale en français, Paris, L'Harmattan.

LEFEUVRE F., 2006a, Quoi de neuf sur quoi ? Etude morphosyntaxique du mot quoi, Rennes, Presses universitaires de Rennes.

LEFEUVRE F., 2006b, « La structure en de quoi », Journal of French Language Studies, 16, p. 51-68.

LEFEUVRE F., 2007, « Le segment averbal comme unité syntaxique textuelle », in Charolles M., Fournier N., Fuchs C. \& Lefeuvre F. (eds), Parcours de la phrase, Ophrys, p. 143-158 (halshs-00138297). 
Lefeuvre F. \& Pierrard M., 2008 : «Les Proformes indéfinies, Indéfinition et prédication », Langue française 158 (Lefeuvre F. \& Pierrard M. eds), p. 3-12.

LeONARDUZZi L., 2004, La Subordonnée interrogative en anglais contemporain, Aix-enProvence, Publications de l'Université de Provence.

Martin R. et WiLmet M., 1980, Syntaxe du moyen français, Bordeaux, Bière.

MARTIN R., 1985, «L'interrogation comme universel du langage », L'interrogation (Valentin ed), Presses de 1'Université de Paris-Sorbonne, p. 257-284.

Meillet A. \& VendRyes J., 1960, Traitement de grammaire comparée des langues classiques, Paris, Champion.

Moline E., 2009, « La norme et l'usage. Approche d'une évolution générationnelle : les exclamatives en comment $»$, Cahiers de l'Asdifle $\mathrm{n}^{\circ} 20$.

Moline E., soumis a «De l'interrogation à l'exclamation: comme $P$ argument de savoir $\mathrm{XVII}^{\mathrm{e}}-\mathrm{XX}^{\mathrm{e}}$ siècles ».

Moline E., soumis a « Vous savez comme j’ai résisté depuis Gand, Scalarité et interprétation de comme $P$ argument de savoir », Hadermann, Pierrard, Van Raemdonck eds.

Muller C., 1996, La Subordination en français, Paris, Colin.

PIERRARD M., 1988, La relative sans antécédent en français moderne, Louvain, Peeters.

PIERRARD M., 1998, « Comme « relatif à antécédent » en ancien français : grammaticalisation de la proforme indéfinie », Travaux de linguistique, n³6, p. 127-146.

Pierrard M. et LEARD J.-M., «2004, Comme : comparaison et haut degré », Intensité, comparaison, degré, Travaux linguistiques du CerLiCO (Lefeuvre et Noailly eds.), 17, p. 269-286.

SANDFELD Kr., 1965, Syntaxe du français contemporain, Les propositions subordonnées, Genève, Droz.

SERBAT G., 1985, «Le verbe introducteur de la subordonnée interrogative en latin », L'Interrogation (Valentin ed.), Presses de l'Université de Paris-Sorbonne, p. 3-16.

WArtburg (von) W. et ZUMTHOR P., 1947, 4e éd (1989), Précis de syntaxe du français contemporain, Berne, Francke.

WILMET, M., 2003, Grammaire critique du français, Bruxelles, Duculot. 